\title{
Une analyse théorique des fondements et du fonctionnement \\ de la relation d'autorité intrafirme
}

(octobre 2002) à paraître dans la Revue Economique

Bernard Baudry*

Bruno Tinel**

Depuis une vingtaine d'années, le thème de l'autorité dans la relation d'emploi a fait l'objet d'un regain d'intérêt parmi les économistes, en raison notamment de la prise en compte de l'incomplétude des contrats. Dans cet article, notre objectif est double. Il s'agit tout d'abord de porter un jugement sur la fameuse controverse Coase / Alchian-Demsetz, en étudiant les fondements de l'autorité intrafirme. Il s'agit également de montrer que la relation d'autorité intrafirme ne peut se résumer à une variable unique, mais au contraire repose sur une pluralité de mécanismes. Dès lors, compte tenu de cette pluralité, la relation d'autorité entre un employeur et un employé doit s'appréhender en termes de degré, et non en termes d'autorité infinie -ou nulle- de l'employeur sur l'employé.

Mots-clés : autorité, firme (théorie de la), contrats incomplets, économie du droit.

FOUNDATIONS AND FUNCTIONING OF AUTHORITY IN FIRMS : A THEORETICAL ANALYSIS

For about twenty years, the notion of authority has been put forward again into the analysis of employment relationship, especially concerning contracts incompleteness. This article has two purposes. First, it aims at giving an assessment of the famous controversy between Coase and Alchian-Demsetz through an analysis of the foundations of authority. Second, it is shown that the authority relationship inside the firm cannot only depend on a single variable. On the contrary, authority rests upon a multiplicity of mechanisms. Employer's authority over employee has therefore to be understood in terms of degrees rather than in boolean terms.

Keywords : authority, firm (theory of the), incomplete contracts, law and economics.

Classification JEL : B210, D230, J530, L220, M500.

\footnotetext{
* Université Lumière Lyon 2, 16 quai Claude Bernard 69365 Lyon Cedex 07, Centre Walras (CNRS - UMR 50 56), bernard.baudry@univ-lyon2.fr.

** Université Paris 1 Panthéon-Sorbonne, MATISSE (CNRS - UMR 85 95), MSE 106-112 boulevard de l'Hôpital, 75647 Paris cedex 13, btinel@univ-paris1.fr (B. Tinel était membre du Centre Walras lorsque le présent article a été rédigé). très utiles.

Que soient remerciés Pierre Dockès, Antoine Jeammaud et les deux rapporteurs dont les commentaires ont été
} 
Dans son ouvrage Les limites de l'organisation, Kenneth Arrow consacre tout un chapitre au rôle de l'autorité dans les organisations. Il écrit ainsi : «la prédominance de l'affectation autoritaire est l'une des caractéristiques les plus répandues des organisations. De manière presque universelle, quelle que soit la taille de l'organisation, des décisions sont prises par quelques individus et exécutées par d'autres » (Arrow [1976], p. 75). Dans ce chapitre, Arrow insiste notamment sur la valeur de l'autorité comme dispositif de coordination au sein des organisations, lorsque les intérêts et/ou l'information diffèrent entre les membres de l'organisation. L'analyse de l'autorité par Arrow, si elle présente l'intérêt de mettre l'accent sur l'autorité comme dispositif de coordination, n'est pour autant pas pionnière puisque des travaux importants sur le thème de l'autorité intraorganisation, et donc intrafirme, ont précédé ce point de vue, tant du côté des théoriciens des organisations ${ }^{1}$ que des économistes.

Du côté des économistes, deux travaux, parmi les plus cités, se distinguent, l'article de Ronald Coase de 1937 d'une part, l'article d'Armen Alchian et d'Harold Demsetz de 1972 d'autre part. D'une certaine manière, le deuxième article constitue une réponse au premier, dans la mesure où pour Alchian et Demsetz, et à l'opposé de l'analyse de Coase qui considère que l'autorité constitue le critère distinctif de la firme par rapport au marché, l'autorité ne permet pas d'effectuer cette opposition, l'employeur pour ces deux auteurs ne disposant pas vis-àtvis d'un employé de plus d'autorité qu'un client vis-à-vis de son épicier. Cet article, niant donc toute forme d'autorité qui serait spécifique à la firme, a dès lors constitué le point de départ d'une série de travaux sur le thème de la nature de la firme et de l'autorité intrafirme, de la part d'économistes soucieux d'analyser les mécanismes de coordination internes à la firme.

Dans les années soixante-dix, la reconnaissance de l'incomplétude des contrats en général, et, pour ce qui est de l'autorité intrafirme, de l'incomplétude du contrat de travail a relancé le débat autour du concept d'autorité. Cette reconnaissance, initiée par les travaux de Williamson, et notamment par son ouvrage de 1975, ouvre en effet la voie à la recherche de mécanismes susceptibles de combler les vides du contrat en cours d'exécution et ex post, lorsque des divergences et des conflits apparaissent entre les contractants par rapport au contrat conclu ex ante. La fonction de l'autorité, en lien avec d'autres mécanismes, et notamment des mécanismes incitatifs, est alors clairement de combler ces vides contractuels.

À cet égard, il faut citer la contribution de Claude Ménard [1994] qui vise à établir une nette distinction entre les notions d'autorité et de hiérarchie, l'une et l'autre étant considérées comme deux

\footnotetext{
1 En ce qui concerne ceux-ci, nous mentionnerons dans cette introduction deux auteurs, Chester Barnard et Max Weber. Ils proposent en fait deux conceptions différentes de l'autorité, Barnard développant plutôt une conception
} 
formes différentes de la notion plus large de commandement, toutefois nous n'aborderons pas ici une telle problématique, et seule la notion d'autorité, telle que définie par Herbert Simon dans son article de 1951 sera abordée dans ce texte ${ }^{2}$.

Nous nous intéresserons plus particulièrement dans ce texte à la question suivante, posée par Oliver Hart dans son ouvrage de 1995 : pourquoi, au sein de la firme, un individu, en l'occurrence le salarié, obéit-il à la personne investie d'une autorité, c'est-à-dire l'employeur ? (p. 57). Plus loin, Hart, faisant allusion à l'article d'Alchian et Demsetz de 1972, note à juste titre qu'une entreprise indépendante peut également donner des ordres à une autre entreprise (idem). La question, centrale par rapport à la controverse Coase versus Alchian-Demsetz, est donc bien de savoir pourquoi un salarié obéit, alors qu'une entreprise indépendante n'obéit pas nécessairement.

Par rapport à cette interrogation, notre objectif est double. Il s'agit tout d'abord, en mobilisant des travaux que nous considérons comme majeurs, de porter un jugement sur cette controverse, en étudiant les fondements de l'autorité intrafirme. Ceux-ci permettent-ils de différencier la relation d'emploi de la relation d'achat-vente ? Au-delà, nous souhaitons montrer dans ce texte que la relation d'autorité intrafirme, relation complexe s'il en est, ne peut se résumer à une variable unique, mais au contraire repose sur une pluralité de mécanismes. Dès lors, compte tenu de cette pluralité, nous pensons qu'en définitive la relation d'autorité entre un employeur et un employé doit s'appréhender en termes de degré, et non en termes d'autorité infinie -ou nulle- de l'employeur sur l'employé.

Compte tenu de cette problématique, nous adopterons le plan suivant. Dans une première section, nous analyserons ce qui nous semble constituer les fondements de la relation d'autorité. Ceux-ci sont à rechercher dans la spécificité de la relation d'emploi, à la fois rapport de pouvoir et rapport contractuel juridique singuliers entre l'employeur et l'employé. Rapport de pouvoir tout d'abord, dans la mesure où cette relation, contrairement à une relation d'achat-vente entre deux contractants indépendants, met face à face deux propriétaires d'actifs différents mais complémentaires, le propriétaire des actifs non humains et le propriétaire des actifs humains. La théorie des droits de propriété, avec notamment les travaux de Hart, et Hart et Moore, constitue de ce point de vue une référence essentielle. Rapport contractuel encadré par des règles juridiques particulières ensuite, puisque le contrat de travail consacre la subordination juridique de l'employé. Pour un auteur

\footnotetext{
consensuelle et horizontale, Weber, pour sa part, à travers son étude du modèle bureaucratique, insistant inversement sur le caractère vertical de l'autorité, autorité fondée notamment sur la compétence mais aussi sur les règles légales.

2 Pour Simon, si l'on définit x comme un élément de l'ensemble de tous les comportements possibles effectués par un salarié dans le cadre de son emploi, «nous dirons que B [le patron, boss] exerce une autorité sur W[1'employé, worker] si $W$ autorise $B$ à sélectionner $x$. Par conséquent, $W$ accepte l'autorité lorsque son comportement est déterminé par la décision de $B »$ (Simon [1951], p. 294).
} 
comme Masten, la jurisprudence montre clairement des différences substantielles entre le régime juridique du contrat de travail et celui du contrat commercial. L'étude des fondements de la relation d'autorité nous permettra alors de porter un jugement sur la controverse Coase / Alchian-Demsetz. Mais, comme nous le verrons dans la deuxième section, l'étude des fondements, des sources de la relation d'autorité n'épuise pas la question de l'autorité dans la firme. En effet, le fonctionnement de cette relation fait apparaître deux autres mécanismes, présents à des moments différents de la relation d'emploi. Comme le soulignent à la fois Rajan et Zingales et Aghion et Tirole, l'employé est tout d'abord à même de mobiliser certaines ressources dans le cours de la relation d'emploi, ressources issues de la spécificité de ses actifs humains. Le niveau de ces ressources modifie alors le rapport de pouvoir entre l'employeur et l'employé, donc le degré d'autorité. Par ailleurs, les travaux de Simon, de Williamson et de Kreps montrent que la relation employeur / employé, au moins au moment de la conclusion du contrat, ressortit d'un engagement implicite de l'employé qui accepte volontairement la relation d'autorité. Ici encore, le niveau d'acceptation a un impact sur le degré d'autorité entre l'employeur et l'employé.

Nous serons alors à même de proposer en conclusion un schéma global d'explication de la relation d'autorité intrafirme, en insistant sur la pluralité des mécanismes qui la régissent. Cette pluralité détermine finalement le degré d'autorité entre un employeur X et un employé Y.

\section{SECTION I. LES FONDEMENTS DE LA RELATION D'AUTORITE : PROPRIETE DES ACTIFS NON HUMAINS ET REGLES JURIDIQUES}

Les fondements de la relation d'autorité intrafirme se situent selon nous à deux niveaux distincts mais complémentaires : ils résultent de l'effet combiné de la propriété des actifs non humains, laquelle propriété conduit au pouvoir de l'employeur sur l'employé (I), et de la règle juridique qui fait naître de tout contrat de travail un lien de subordination de l'employé vis-à-vis de son employeur (II).

\section{I/ Propriété des actifs non humains, pouvoir et relation d'autorité}

La propriété des actifs est placée au centre de l'analyse par la théorie des contrats incomplets, appelée aussi théorie des droits de propriété, dont le principal modèle est issu des contributions de Grossman et Hart [1986], Hart et Moore [1990] et Hart [1995]³. Au delà de ses particularités

\footnotetext{
3 Nous écrirons par la suite GHM pour Grossman, Hart et Moore. Cette approche reprend de manière plus rigoureuse et généralise le cadre d'analyse préexistant du "hold-up", développé initialement autour de la notion de quasi-rente appropriable par Williamson [1975], Golberg [1976] et Klein, Crawford \& Alchian [1978] ; pour une revue récente de
} 
formelles, cette approche développe une réflexion originale sur la signification économique de l'incomplétude contractuelle. Sans nier l'importance des coûts de transaction, GHM s'intéressent en premier lieu à l'idée d'incomplétude en lien avec celle de propriété d'un actif. Il s'agit pour ces auteurs de cerner précisément les effets incitatifs de la propriété. Les contrats -incomplets- ne pouvant, par définition, spécifier toutes les utilisations d'un actif donné en fonction de tous les états du monde, il devient important de savoir qui dispose du droit de décider de l'usage de cet actif dans les cas où le contrat ne donne pas d'instruction précise. La théorie des droits de propriété octroie ce droit au propriétaire de l'actif en vertu de l'idée que la possession donne des droits résiduels de contrôle. Il s'agit du droit de décider de tous les usages de l'actif tant que ceux-ci ne sont pas incompatibles avec les contrats signés antérieurement, avec la coutume ou avec la loi. Schématiquement, la problématique de la théorie des droits de propriété consiste à montrer que les individus sont plus enclins à développer des investissements relationnels spécifiques lorsqu'ils possèdent les actifs nécessaires à la valorisation de leur effort productif. La propriété d'un actif confère à son titulaire le privilège de décider de son utilisation, à travers les droits résiduels de contrôle. En particulier, il peut exclure à discrétion les autres individus de l'utilisation de cet actif. Pour GHM, la notion de droits résiduels de contrôle d'un actif, cette faculté d'exclusion discrétionnaire conférée par la propriété, est identique à celle de pouvoir ${ }^{4}$. Etant donnée cette identité, le pouvoir est nécessairement indirect : tout pouvoir passe par la propriété d'un actif5.

Quelles sont les implications de cette théorie en ce qui concerne l'analyse de la relation d'emploi? Reprenant la célèbre discussion d'Alchian et Demsetz [1972] sur la source incertaine de l'autorité, où ceux-ci comparaient la relation employeur-employé à celle que nouent un épicier et son client, Hart suggère, comme nous l'avons déjà indiqué dans l'introduction, que la question pertinente est la suivante : pourquoi un employé prête-t-il probablement plus attention à ce que lui ordonne son employeur que ne le fait un contractant indépendant ? «Dans le premier cas, répond-il, si la relation s'arrête, l'employeur part avec tous les actifs non-humains, en revanche dans l'autre cas

la littérature sur ce thème, cf. Malcomson [1997], bien qu'il ne fasse étrangement aucune allusion à ces articles fondateurs. Pour une synthèse formalisée sur les derniers développements de la théorie des contrats incomplets, le lecteur consultera la Review of Economic Studies, numéro spécial de janvier 1999. Enfin, pour une évaluation méthodologique, à l'aune des contrats complets, de l'apport de la théorie des contrats incomplets, le lecteur consultera la contribution de Tirole [1999]; pour une critique des principaux résultats des modèles à la GHM, cf. Holmström [1999] et Holmström \& Roberts [1998].

4 Dans l'introduction de son ouvrage de 1995, Hart emploie en effet de façon interchangeable les vocables "control" et "power". Le terme de pouvoir, qui revient une vingtaine de fois entre les pages 3 et 6, désigne donc les droits résiduels de contrôle.

5 Toutefois, la propriété peut ne pas conférer de pouvoir si les contrats sont complets. Dans un monde où $\mathbf{1}$ est réalisable de signer de tels contrats, tous les possibles peuvent être envisagés à l'avance : aucun individu ne peut donc bénéficier d'un quelconque droit résiduel de contrôle, c'est à dire d'un quelconque pouvoir. Dans ce cas, la propriété ne confère pas de pouvoir, elle apporte uniquement un revenu spécifique. 
chaque contractant indépendant conserve des actifs non-humains. L'individu i est davantage susceptible de faire ce que veut l'individu j si j peut exclure i des actifs dont i a besoin pour être productif que si i peut garder ces actifs avec lui(...). En d'autres termes, le contrôle des actifs nonhumains mène au contrôle des actifs humains » (Hart [1995], p. 58, souligné par l'auteur ). Pourquoi le salarié obéit-il à l'employeur? Parce que ce dernier peut le priver de l'usage des moyens de production. L'autorité de l'employeur sur l'employé est donc indirecte car elle découle du pouvoir que confère la propriété. L'employeur n'a pas de pouvoir direct sur l'employé lui-même. Pour résumer, le pouvoir dont dispose un employeur de décider qui peut utiliser les actifs qu'il possède lui confère aussi une autorité sur l'employé, au sens de Simon. Hart montre ainsi que l'autorité 6 dans l'entreprise est fondée sur la propriété des moyens de production

Cette idée d'une relation d'autorité fondée sur la propriété des actifs non-humains comporte certaines hypothèses qu'il convient d'expliciter. En effet, supposer que l'autorité de l'employeur provient de la menace crédible de priver l'employé des actifs non humains s'il n'obéit pas, implique à la fois 1) que les actifs non-humains de l'employeur soient indispensables à l'employé pour que ce dernier soit productif et donc obtienne un revenu 8 et, d'autre part, 2) que les actifs humains de l'employé ne soient pas quant à eux indispensables pour l'employeur. Par conséquent, "dès le départ", il n'y a pas de symétrie entre l'employé et l'employeur puisque l'un est dépendant économiquement de l'autre. Ainsi, l'employé dispose de peu d'alternatives en dehors de sa relation spécifique avec son employeur (le propriétaire des moyens de production ou son représentant) pour valoriser son effort productif. Par conséquent, sa force de travail n'est pas redéployable sans coûts importants. Qu'en est-il pour l'employeur? Il n'est pas dépendant de son employé car, d'une part, si la relation cesse, il ne perd pas la totalité de son revenu et, d'autre part, il cherchera à organiser la production de manière à pouvoir le remplacer pour un faible coût (cf. section II, I/, A/).

Pour résumer, les droits résiduels de contrôle rendent compte d'un pouvoir sur les choses conféré par la propriété, et ce pouvoir sur les choses donne de l'autorité sur les hommes. L'employé obéit à

\footnotetext{
6 Toutefois, la théorie des contrats incomplets ne donne pas d'indication sur le fonctionnement même de la relation d'autorité, ainsi que l'écrivent Hart et Moore [1990] en conclusion de leur article : «dans notre modèle, un employeur n'a jamais à dire à un employé ce qu'il doit faire : simplement, celui-ci l'imagine par lui-même et agit en conséquence » (p. 1153).

7 On remarquera que cette application par Hart de la notion de quasirente relationnelle à la relation d'emploi n'est pas étrangère aux arguments invoqués par Shapiro \& Stiglitz [1984] pour expliquer l'existence d'un chômage involontaire.

8 En termes marxiens, le travailleur est contraint de vendre sa force de travail pour obtenir un revenu. Notons à ce propos que Hart considère que sa préoccupation relative à l'allocation du pouvoir dans les relations contractuelles n'est pas étrangère à la problématique marxienne: "Étant donné son rapport avec la question du pouvoir, l'approche proposée dans cet ouvrage a quelque chose en commun avec les théories marxiennes de la relation travailleurcapitaliste, en particulier avec l'idée qu'un employeur a du pouvoir sur un travailleur parce qu'il possède le capital physique qu'utilise le travailleur (et donc peut s'approprier le surplus du travailleur). La connexion entre les deux approches n'a malgré tout pas encore été développée dans la littérature » (Hart [1995], p. 5, n. 5).
} 
l'employeur, ou devance ses souhaits dans certaines limites, 1) parce qu'il possède uniquement ses actifs humains et 2) parce qu'il a besoin d'utiliser les actifs non humains que contrôle l'employeur pour obtenir un revenu. L'employé est donc a priori économiquement dépendant de l'employeur. L'autorité de celui-ci repose sur la menace crédible de licenciement que produit cette dépendance. Toutefois, cette approche surestime le pouvoir de l'employeur et demeure très incomplète. D'une part, elle ne rend pas compte du fonctionnement effectif de l'autorité car elle néglige totalement les ressources à la disposition de l'employé lui permettant de limiter le pouvoir de l'employeur et, de plus, elle ignore l'aspect coopératif de la relation d'emploi 9 . D'autre part, elle délaisse l'environnement institutionnel dans lequel s'insère la relation d'autorité, objet du point suivant.

\section{II/ Les fondements institutionnels de la relation d'autorité : la subordination juridique}

$\mathrm{Si}$, comme nous venons de le voir, la propriété des actifs non humains procure à l'employeur un pouvoir de direction des employés, pouvoir qui conduit à l'autorité, elle ne constitue pas le seul fondement de cette dernière. En effet, le droit, via un certain nombre de règles, consacre la subordination de l'employé, et donc l'autorité de l'employeur, en imposant à l'employé des contraintes, des obligations.

Dans ce registre, l'article de 1988 de Scott Masten, consacré à la nature de la firme, est selon nous exemplaire ${ }^{10}$. Masten, dans ce texte, s'attache à identifier les éléments qui permettent selon lui de différencier la firme du marché, en décortiquant les dispositifs juridiques et la jurisprudence consacrés au contrat de travail et au contrat d'achat-vente. En utilisant ce registre, Masten fait indiscutablement figure d'économiste quelque peu atypique, et d'ailleurs il souligne lui-même cette étrange éviction de l'environnement institutionnel et donc des règles juridiques par la plupart des économistes s'intéressant à la nature de la firme. Il cite ainsi l'article célèbre de Jensen et Meckling de 1976, dans lequel ces deux auteurs considèrent la firme comme un "noeld de contrats", et donc qu'il est inutile de vouloir distinguer les éléments qui sont internes à la firme de ceux qui lui sont extérieurs. Mais Masten indique que ces deux auteurs, curieusement, soulignent pourtant, certes dans une note de bas de page, l'importance du système juridique : « Cette vision de la firme [comme un noud de relations contractuelles] met en avant le rôle important que jouent le système juridique et la loi dans les organisations sociales, particulièrement dans l'organisation de l'activité économique. »(Jensen \& Meckling [1976], p. 311). Reprenant finalement à son compte cette problématique soulignée par Jensen et Meckling mais au bout du compte évincée, Masten se pose la

\footnotetext{
${ }^{9}$ Ces deux points sont abordés en section II.

10 Voir également en français l'article de Jeammaud et al. [1998].
} 
question suivante : «la loi traite-t-elle, par exemple, d'une manière équivalente les transactions commerciales et d'emploi?» (Masten [1991], p. 199). Pour répondre à cette question, sa démonstration s'effectue en deux temps ${ }^{11}$. Nous résumerons brièvement sa démarche et ses résultats, puis nous porterons une appréciation critique sur son travail.

Tout d'abord, l'auteur se demande s'il existe des droits et une autorité conférés à l'employeur qui ne seraient pas utilisables dans une relation d'échange purement commercial, c'est-à-dire entre des entreprises indépendantes. Au terme de cette première investigation, il souligne avec force que la loi ne traite pas de manière équivalente une relation commerciale et la relation d'emploi: «l'investigation révèle que la loi reconnaît en fait des différences substantielles dans les obligations, les sanctions et les procédures régissant les deux types d'échange » (ibid., p. 208). En ce qui concerne plus précisément l'autorité, Masten souligne que lorsqu'un individu s'engage dans une relation d'emploi, il accepte «une obligation tacite de faire preuve d'obéissance à toutes les règles, les ordres et les instructions raisonnables de l'employeur » (ibid., p. 199). Plus précisément, pour les tribunaux américains, dans la relation d'emploi, l'autorité, et le contrôle qui s'y rattache, porte non seulement sur le résultat du travail, mais aussi sur la manière dont le travail est effectué. En revanche, et c'est là une différence fondamentale, dans une transaction commerciale, le contrôle ne peut porter que sur le résultat du travail ${ }^{12}$. Et Masten de conclure : «donc, l'insistance traditionnelle en économie sur l'autorité du management pour diriger l'effort des employés est au moins nominalement appuyée par la loi régissant les transactions d'emploi » (ibid., p. 200).

Puis, dans une deuxième étape, essentielle, Masten s'interroge sur l'existence ou non de mécanismes qui autorisent la mise en oevvre effective de l'autorité au sein de la firme. Autrement dit, il s'agit de savoir quels sont les moyens mis à la disposition de l'employeur pour que notamment les ordres soient exécutés par les employés. Or, pour Masten, si le pouvoir de l'employeur réside uniquement dans la menace du licenciement, alors son autorité n'est guère différente de celle d'un contractant indépendant engagé dans une relation d'échange commercial, même si par ailleurs il existe un devoir formel d'obéissance de la part de l'employé. En effet, la possibilité d'interrompre à tout moment un flux d'échange commercial constitue également la seule sanction normalement utilisable par un contractant indépendant qui n'est pas satisfait de son cocontractant. Autrement dit, et ceci est un aspect crucial du point de vue de la nature de l'autorité intrafirme, cette dernière ne

\footnotetext{
11 Dans son étude, Masten ne s'intéresse pas seulement à la question de l'autorité, il analyse également, toujours d'un point de vue juridique, d'autres propriétés du contrat de travail par rapport au contrat commercial, et notamment sa flexibilité et sa capacité à réduire l'asymétrie informationnelle existante entre l'employeur et l'employé. Seule la première question retiendra notre attention.

12 On notera au passage que l'on retrouve ici la définition de la relation d'emploi telle qu'elle est appréhendée par Coase dans son article de 1937.
} 
peut pas seulement être fondée, pour Masten, sur la seule menace de la rupture du contrat de travail, car sinon, comme le soulignent Alchian et Demsetz dans leur article de 1972, il n'existe pas une autorité qui serait spécifique à la firme, et plus précisément à la relation d'emploi.

Masten va alors s'attacher dans cette deuxième étape à montrer, toujours à partir de la jurisprudence américaine, que le licenciement ne constitue justement pas la seule sanction à la disposition de l'employeur en cas de manquement à ses obligations par un employé. En effet, dans ce cas de figure, l'employé doit faire face à des dommages et intérêts si ces manquements causent du tort aux affaires de l'employeur. Autrement dit, alors que la conduite et la loyauté des participants engagés dans une transaction commerciale sont strictement une question de jugement, la loi oblige l'employé à agir dans l'intérêt de son employeur (Masten [1991], p. 202) ${ }^{13}$. Masten est alors en mesure de conclure de la manière suivante sa démonstration : «Le point important est que le droit habilite un employeur à obtenir des dommages-intérêts d'un subordonné déloyal ou non-coopératif et donc différencie les incitations des employés de celles des contractants indépendants d'une manière discrète, modifiant la rémunération du comportement non-coopératif de l'employé d'une façon dont on peut soutenir qu'elle supporte l'autorité communément attribuée dans la théorie de la firme aux employeurs » (ibid., p. 203). Dès lors, et ceci est pour Masten en contradiction avec la démonstration d'Alchian et Demsetz, les effets de la loi sont tels que l'employé qui souhaite préserver son contrat de travail a de plus fortes incitations à satisfaire les ordres de son employeur donc à accepter l'autorité de ce dernier-, qu'un contractant commercial vis-à-vis d'un autre transactant.

En définitive, il est possible de synthétiser l'analyse de Masten, et au-delà l'apport du droit ${ }^{14}$, de la manière suivante.

Tout d'abord, les règles juridiques institutionnalisent le pouvoir de direction économique de l'employeur, pouvoir issu de la propriété des actifs non humains. Le droit garantit ainsi à l'employeur la liberté d'utilisation de ses actifs non humains (création d'activité, organisation de la production).

Ensuite, les règles juridiques offrent des ressources complémentaires aux ressources procurées par la propriété des actifs non humains (pouvoir de direction des personnes), en consacrant la subordination juridique de l'employé, par l'intermédiaire du contrat de travail. C'est ainsi que l'employeur est libre, bien évidemment dans les limites définies par la loi, de "compléter" le contrat de travail, en fonction des aléas qui surgissent dans le cours de la relation d'emploi.

\footnotetext{
13 A noter qu'il en est de même en France, puisqu'«une certaine loyauté envers l'entreprise s'impose au salarié» (Jeammaud et al. [1998], p. 363).

${ }^{14}$ Cf. Jeammaud et al., op. cit.
} 
Enfin, les règles juridiques procurent à l'employeur d'autres sanctions que celles issues de la seule propriété des actifs non humains.

En revanche, nous nous écartons de l'analyse de Masten sur un point essentiel, qui a trait à l'opposition entre relation d'emploi et relation d'achat- vente. En effet, contrairement à Masten, nous pensons que la sanction consistant à mettre fin à la relation, que ce soit de la part d'un employeur ou de la part d'un client, ne sont pas équivalentes dans la mesure où les conséquences de cette cessation sont différentes dans les deux cas : dans le cas de deux contractants indépendants, chaque contractant conserve ses propres actifs non humains, et donc la possibilité de continuer ses affaires. Au contraire, dans le cas de la relation d'emploi, comme nous l'avons vu avec la thèse de Hart, le salarié conserve uniquement ses actifs humains, ce qui procure à l'employeur un pouvoir, pouvoir que ne possède pas le contractant indépendant ${ }^{15}$. En ce sens, dire que l'autorité intrafirme ne peut pas seulement être fondée sur la menace de la rupture du contrat de travail, car sinon, il n'existerait pas une autorité qui serait spécifique à la relation d'emploi -comme le soulignent Alchian et Demsetz-, c'est nier ce qui fait la spécificité de la relation d'emploi, c'est-à-dire la présence d'actifs non humains qui sont indispensables à l'employé pour être productif (voir supra). La nature de cette relation est donc tout à fait différente de la relation entre un épicier et son client, ou d'une relation entre deux entreprises indépendantes, puisqu'aucun actif n'est attaché à cette deuxième relation.

$\mathrm{Au}$ total, cet article de Masten témoigne de la richesse d'un véritable travail pluridisciplinaire et contribue à souligner l'importance de l'environnement institutionnel sur le choix des structures de gouvernement retenues par les contractants, élément ignoré par la plupart des économistes qui s'intéressent à la question de l'autorité intrafirme. On rejoint ainsi le point de vue formulé par Thierry Kirat selon lequel «le choix d'une forme d'organisation est, entre autres, fonction des ressources qu'offrent les corps de règles applicables à la forme choisie » (Kirat [1998], p. 1081).

$\mathrm{Au}$ terme de cette première section, nous sommes en mesure de porter un jugement sur la controverse Coase / Alchian-Demsetz, à propos de l'autorité. En effet, l'étude des fondements de l'autorité intrafirme montre clairement des différences entre la relation d'emploi et une relation commerciale entre des contractants indépendants. Ces différences expliquent pourquoi le salarié obéit alors même que le contractant indépendant n'obéit pas nécessairement. D'une part, dans le cas de la relation d'emploi, en cessant la relation, le propriétaire des actifs non humains prive l'employé de l'accès à ces actifs, accès indispensable à la perception d'un revenu, alors que dans le cas d'une

\footnotetext{
15 Sauf bien évidemment dans le cas peu probable où un contractant indépendant dépendrait pour son chiffre d'affaires à $100 \%$ d'un autre contractant. Notons par ailleurs que dans ce cas de figure, la relation serait vraisemblablement requalifiée en relation d'emploi.
} 
transaction commerciale, chaque individu conserve ses actifs non humains ${ }^{16}$. D'autre part, les règles juridiques applicables à la relation d'emploi sont différentes de celles qui régissent la relation commerciale, en termes d'obligations et de sanctions, et elles consacrent l'autorité de l'employeur, contrairement à la relation commerciale où les contractants sont sur le même pied d'égalité juridique.

\section{SECTION II. LE FONCTIONNEMENT DE LA RELATION D'AUTORITE : ACTIFS SPECIFIQUES HUMAINS ET CONTRAT IMPLICITE}

Analyser les fondements de l'autorité intrafirme ne suffit pas seulement selon nous à la caractériser; en effet, le fonctionnement de cette relation fait apparaître deux mécanismes qui vont influer sur le degré d'autorité dont dispose l'employeur sur l'employé, la particularité de ces deux mécanismes étant qu'ils se situent à deux moments différents de la relation d'emploi. Il s'agit tout d'abord des ressources que possède l'employé, ressources susceptibles de contrebalancer le pouvoir de l'employeur issu de la possession des actifs non humains. Ces ressources sont constituées par les actifs spécifiques humains que l'employé est en mesure de développer en cours de contrat. Le deuxième mécanisme renvoie à la dimension coopérative de la relation d'emploi : au moment de la conclusion du contrat, l'employé adhère librement à l'organisation dans laquelle il rentre, et donc il accepte en quelque sorte l'autorité de l'employeur.

\section{I/ Actifs spécifiques humains, pouvoir de l'employé et relation d'autorité}

L'analyse, à elle seule, des rôles respectifs de la propriété des moyens de production et de l'environnement institutionnel dans la relation d'emploi peut laisser croire que l'employé ne dispose d'aucune ressource, interne à la relation, pour limiter l'autorité de l'employeur. Les contributions majeures de Rajan et Zingales [1998] et Aghion et Tirole [1997] tentent de remédier à cette insuffisance. Sans pour autant prétendre bien évidemment que la relation d'emploi soit parfaitement équilibrée (le déséquilibre en faveur de l'employeur est plutôt considéré dans cette littérature comme le garant de l'efficience), ces auteurs considèrent que l'autorité de l'employeur sur l'employé est circonscrite par deux éléments principaux : la spécificité des actifs humains et les asymétries informationnelles.

\footnotetext{
16 On pourrait objecter à ce raisonnement que le contractant indépendant perd également l'accès à un client, mais,
} comme nous l'avons indiqué dans la note précédente, il est extrêmement rare qu'un contractant indépendant dépende 


\section{$\underline{\text { A/ Le rôle de la spécificité des actifs }}$}

L'employé dispose, dans certains cas, d'une ressource importante pour limiter le degré de l'autorité de l'employeur : son capital humain. Toutefois, celui-ci ne confère un pouvoir à son détenteur que s'il est suffisamment valorisable. Rajan et Zingales développent cette idée à partir de la notion "d'accès", lequel se définit comme «la capacité d'utiliser, ou de travailler avec, une ressource critique. Si la ressource critique est une machine, l'accès implique la capacité à faire fonctionner la machine; si la ressource est une idée, l'accès implique d'être exposé aux détails de cette idée; si la ressource est une personne, l'accès consiste en l'aptitude à travailler étroitement avec cette personne. L'agent qui obtient un accès aux ressources obtient (...) l'opportunité de spécialiser son capital humain avec ces ressources et de se rendre lui-même valorisable » (Rajan \& Zingales [1998], p. 388) ${ }^{17}$. L'accès influe sur l'équilibre des forces entre l'employeur et l'employé à deux niveaux. D'une part, il conditionne le potentiel de spécialisation de l'employé et, d'autre part, ce potentiel est plus ou moins grand selon le degré d'exclusivité de l'accès.

L'idée centrale, absente chez GHM, est que la propriété peut ne pas inciter à fournir un effort productif conséquent. En effet, la spécialisation dans certaines tâches peut détruire les options extérieures du propriétaire. Dans ces cas, donner l'accès aux actifs non humains à un tiers, un employé, sera plus intéressant car "l'insécurité [due à l'absence de propriété] peut encourager plutôt que décourager l'investissement spécifique des parties prenantes » (ibid., p. 424, souligné par les auteurs). ${ }^{18}$ L'idée est donc simple et connue : l'accès à certaines ressources critiques permet à l'employé de spécialiser son capital humain par un processus d'apprentissage. Ce faisant, les actifs humains de l'employé acquièrent des caractéristiques spécifiques plus ou moins complémentaires avec les actifs non humains de l'employeur. Cette spécialisation peut rendre

\footnotetext{
d'un seul client pour son chiffre d'affaires, alors que la quasitotalité des salariés dépend, pour son revenu, d'un employeur unique.

17 Rajan et Zingales ne donnent pas de définition précise à la notion, cruciale dans leur raisonnement, de "ressource critique". Malgré son aspect suggestif sur le plan intuitif, la clarification de cette idée permettrait de savoir plus précisément qui est en mesure, parmi les salariés d'une entreprise, de développer des actifs spécifiques susceptibles de leur conférer, à terme, un certain pouvoir individuel.

18 Dans une situation mettant en scène deux individus, C et S, Rajan et Zingales montrent que si l'investissement spécifique de $S$ réduit la valeur de l'actif dans ses autres usages que ceux où $C$ et $S$ interagissent, alors, $S$ investira moins s'il est propriétaire de l'actif. Cet effet désincitatif de la propriété résulte du fait que $S$ préférera se ménager des options extérieures parce qu'il internalise la perte de valeur des alternatives extérieures que produit la spécialisation. Ce principe semble très proche de l'idée défendue par Holmström et Milgrom [1991] pour qui l'une des conditions de l'efficience de la propriété d'un actif est que la variance du rendement de l'actif soit basse. En effet, il est cohérent de poser que la variance du rendement d'un actif est une fonction croissante de la spécialisation, au sens où l'entendent Rajan et Zingales.
} 
l'employé plus ou moins indispensable pour le propriétaire des moyens de production, ce qui rééquilibre le rapport de force ${ }^{19}$.

Les modalités d'exercice du pouvoir par le propriétaire et par le non propriétaire sont symétriques. Le pouvoir du propriétaire d'actifs non humains est crédible lorsqu'il ne risque pas de subir la position de type monopolistique de ses cocontractants. Inversement, le non propriétaire tire son pouvoir uniquement du rapport de force interne à la relation qu'il entretient avec le propriétaire, autrement dit, la spécialisation est une façon pour lui de "brûler ses vaisseaux" en liant son destin à celui de l'actif auquel il a accès, sans pour autant réduire la valeur de ses options extérieures. Dans cette situation proche d'un monopole bilatéral, l'employé dispose donc bien d'un pouvoir limitatif de l'autorité de son employeur mais n'a pas lui même d'autorité sur celui-ci au sens de Simon : il ne lui donne aucun ordre.

Les droits résiduels de contrôle ne sont donc pas seuls générateurs de pouvoir, car l'accès à des actifs (humains ou non) considérés comme des "ressources critiques" pourra permettre aux employés qui en bénéficieront de développer leur capital humain et de se rendre indispensables. 20 Pour cette raison, Rajan et Zingales considèrent l'accès comme une forme de pouvoir. Ce pouvoir de l'employé peut venir limiter l'autorité de l'employeur. Toutefois, cette limitation ne peut survenir que dans un second temps. En effet, dans un premier temps, le pouvoir de décision de l'octroi d'un accès, à tel ou tel actif pour tel ou tel employé, repose entre les mains du propriétaire (ou de son représentant). Le pouvoir limitatif de l'employé est donc subordonné à celui de l'employeur. Les employés qui n'ont pas accès aux ressources critiques ne pourront pas développer les actifs spécifiques susceptibles de leur donner un quelconque pouvoir. Pour ces individus, le “degré" de l'autorité de l'employeur sera fort.

L'accès aux ressources critiques représente alors en lui-même un enjeu lourd de conséquences pour les deux protagonistes car il contribue à conditionner le pouvoir futur de chaque partie. L'employeur aura intérêt à limiter au maximum l'accès à ses actifs critiques pour ne pas subir le pouvoir de ses employés et pour pouvoir les remplacer facilement. Ce choix résultera d'un arbitrage entre perte de contrôle et gains productifs. Inversement, chaque salarié aura intérêt à obtenir un

\footnotetext{
19 Notons ici que l'on trouve cette même idée chez Williamson. Pour ce dernier, les salariés peuvent en effet arbitrer entre des actifs spécifiques à une firme et des actifs généraux. Ceux qui choisissent la deuxième possibilité sont alors en mesure de travailler pour un plus grand nombre d'employeurs (Williamson [1985], p. 259). Pour ceux qui ont choisi la première solution, la menace du licenciement n'est alors plus totalement crédible.

20 A ce propos, Rajan et Zingales insistent sur le fait que «l'agent qui reçoit le privilège de l'accès à une ressource n'obtient aucun nouveau droit résiduel de contrôle. Tout ce qu'il obtient est l'opportunité de spécialiser son capital humain envers la ressource et de se rendre lui-même valorisable. Lorsqu'il est combiné avec le droit résiduel préexistant de retirer son capital humain, l'accès lui donne la capacité de créer une ressource critique qu'il contrôle : son capital humain. Le contrôle sur cette ressource critique est une source de pouvoir» (Rajan \& Zingales [1998], p. 388, souligné par les auteurs).
} 
accès aussi large que possible. Selon quels principes l'employeur décide-t-il de donner un accès à telle ou telle ressource pour tel ou tel individu? La sous-section suivante donnera des éléments de réponse à cette question.

Rajan et Zingales montrent ensuite que le type de technologie productive employée dans l'organisation joue un rôle dans la plus ou moins forte capacité individuelle des employés à limiter l'autorité de l'employeur. Ainsi, une organisation productive reposant sur l'additivité des tâches permet à la fois 1) d'inciter les individus à se spécialiser et 2) de limiter leur pouvoir vis-àt vis de l'employeur. Au contraire, des techniques présentant des caractéristiques de substitution parfaite et de complémentarité entre les tâches ne conduisent à un effort productif suffisant que si elles s'accompagnent d'un droit d'accès exclusif. ${ }^{21}$ En contrepartie, cette exclusivité d'accès octroyée à l'employé lui confère un certain pouvoir vis-à-vis du propriétaire des actifs non-humains. En d'autres termes, chaque fois que les propriétaires d'une firme, ou leurs représentants, ne parviendront pas à appliquer les principes d'une organisation parcellaire du travail, visant à séparer les tâches de manière additive, un arbitrage devra avoir lieu. Soit l'employeur octroiera un droit d'accès exclusif à un petit nombre de salariés, afin de les inciter à se spécialiser suffisamment, soit il ne leur donnera pas ce droit exclusif, au prix d'un output plus faible. L'exclusivité, par le monopole local qu'elle garantit, incite l'employé à se spécialiser davantage et donc à être plus productif, mais en contrepartie elle présente l'inconvénient pour l'employeur d'accroître le pouvoir de l'employé : si l'exclusivité est maintenue, toutes choses égales par ailleurs, la spécialisation augmente les aspects indispensables du capital humain de l'employé. Celui-ci pourra menacer l'employeur d'une privation de l'usage de ses actifs humains. Cette possibilité pour l'employé de se rendre plus ou moins indispensable vient ainsi limiter l'étendue de l'autorité de l'employeur.

Ce raisonnement repose sur une implicite prise en compte endogène de la technologie. En effet, d'une part les caractéristiques techniques du processus de production (additivité, complémentarité ou substituabilité des tâches) peuvent être choisies par l'employeur de manière à limiter plus ou moins le pouvoir de l'employé, d'autre part la spécialisation confère un pouvoir à l'employé parce qu'il peut être conduit, en se spécialisant, à modifier les caractéristiques des actifs non humains. C'est cette modification des actifs, formellement équivalente dans le modèle de GHM à une modification de la technologie, qui réduit la valeur optionnelle extérieure des actifs pour le propriétaire. De quelle manière les actifs non humains vont-ils être modifiées par l'employé ? Celui-

\footnotetext{
21 Par exemple, un tel droit est assuré simplement en ne recrutant qu'une seule personne pour un poste de travail donné, l'enforcement de ce droit dépend ensuite des caractéristiques des autres postes.
} 
ci cherchera à être indispensable pour le propriétaire en rendant les actifs auxquels il a accès idiosyncrasiques à ses propres actifs humains 22.

\section{$\underline{B / L e s}$ asymétries informationnelles}

A ce niveau de l'analyse, la manière dont s'ajustent l'autorité de l'employeur et le pouvoir limitatif de l'employé n'apparaît pas clairement, la situation semble se résumer à un pur conflit. La contribution de Aghion et Tirole [1997] fournit des éléments permettant de comprendre comment se stabilisent ces pouvoirs grâce au mécanisme de délégation de l'autorité. Ils développent un modèle d'allocation de l'autorité formelle et réelle 23 fondé sur les asymétries d'information : l'autorité peut être déléguée d'un supérieur vers son subordonné, étant entendu qu'en dernière instance cette autorité émane de la propriété des actifs ${ }^{24}$. S'inspirant de la discussion de Weber sur la domination rationnelle-légale, dans Economie et Société, ces deux auteurs soulignent que «les fonctionnaires, les employés et les travailleurs attachés au personnel administratif d'une bureaucratie ne possèdent pas eux-mêmes les moyens non-humains de production et d'administration, pourtant ils exercent un contrôle substantiel sur la machine bureaucratique » (Aghion \& Tirole [1997], p. 2). En définissant l'autorité formelle comme le droit de décider et l'autorité réelle comme le contrôle effectif sur les décisions, ces auteurs proposent un modèle où le processus de décision est le suivant : le subordonné, l'agent, propose un "projet" au principal que celui-ci peut soit accepter soit rejeter en fonction de l'information coûteuse qu'il a pu obtenir ${ }^{25}$.

Les bénéfices de la délégation de l'autorité formelle sont de deux types : a) elle augmente l'incitation de l'agent à acquérir de l'information; b) elle augmente sa participation à la relation contractuelle. Du point de vue du principal, le coût de la délégation se traduit par une perte de contrôle dans le choix des projets. Quand faut-il laisser l'autorité formelle entre les mains du principal et quand doit-elle être déléguée à l'agent ? Deux types de déterminants sont à prendre en

\footnotetext{
22 Ce mécanisme par lequel l'employé se rend indispensable en agissant directement sur les caractéristiques des actifs est surtout destiné à rendre compte du pouvoir des managers sur les actionnaires.

23 L'autorité formelle renvoie à la question du droit d'effectuer telle ou telle tâche, l'autorité réelle renvoie à celle l'effectivité de l'exécution.

24 Cette idée d'une détermination de l'autorité formelle en dernière instance par la propriété est réaffirmée avec force par Baker, Gibbons \& Murphy [1999] (BGM) pour critiquer l'idée d'Aghion et Tirole selon laquelle l'autorité formelle pourrait faire l'objet d'une délégation. Pour BGM, toute délégation d'autorité au sein de la firme est forcement informelle. Remarquons contre BGM que l'organigramme de l'organisation constitue bien une formalisation de la délégation de l'autorité.

25 Ce mode d'exercice de l'autorité, que l'on peut qualifier de "passif", rappelle la notion de "droit au dernier mot" dont parle Simon dans Administrative Behavior. Toutefois, contrairement à Simon, Aghion et Tirole tiennent compte du possible décalage entre ce qu'ordonne le supérieur hiérarchique et ce qu'effectue réellement le subordonné.
} 
compte : d'une part, les éléments agissant par le biais de l'initiative et d'autre part, ceux relatifs à la contrainte de participation des agents.

La délégation d'autorité stimule l'initiative de l'agent, c'est à dire l'effort à accomplir ses tâches. En contrepartie, les coûts de la délégation se mesurent évidemment en termes de perte de contrôle réel et formel sur les actions de l'agent. Par conséquent, la délégation d'une tâche ou d'un ensemble de tâches résultera d'un arbitrage entre ces deux éléments. Le rôle de la participation individuelle est mis en évidence à travers un modèle multitâches. Toutes choses égales par ailleurs, la délégation (abstraction faite des phénomènes d'incitations) est adaptée pour les décisions 1) qui comptent peu pour le principal, soit parce qu'elles lui rapportent peu directement, soit parce que l'agent est considéré comme "digne de confiance" et 2) qui sont "importantes" pour l'agent. L'initiative et la participation constituent des déterminants complémentaires de l'allocation de l'autorité formelle. En outre, concernant la propension de l'agent à communiquer l'information pertinente qu'il possède sur les projets, quand la délégation est absente, elle sera transmise plus facilement au supérieur si les objectifs des deux parties sont suffisamment congruents. Si tel n'est pas le cas, la communication sera relativement meilleure dans le cas de la délégation.

Enfin, quels sont les facteurs permettant d'améliorer l'initiative de l'agent sous l'hypothèse que le principal conserve l'autorité formelle ? Tout d'abord, une firme a toujours intérêt à se trouver en situation de surcharge de travail. Ceci correspond au cas où un employé supplémentaire rapporte un profit marginal négatif. La surcharge constitue en effet un engagement crédible de la part du principal à ne pas trop contrôler ses subordonnés et donc à leur laisser une marge d'initiative. Ensuite, la prise en compte de l'effet des incitations monétaires sur le comportement de l'agent indique qu'un salaire plus élevé accroît l'autorité réelle en raison 1) d'une meilleure aptitude à recommander des projets pertinents et 2) d'une réduction de l'incitation du principal à contrôler et donc de la probabilité que l'agent voit ses décisions cassées par son supérieur. Enfin, la multiplicité des principaux, la réputation ${ }^{26}$ du supérieur à n'intervenir qu'en cas extrême et l'urgence de la prise de décision ont des effets positifs sur l'initiative du subordonné.

Ce modèle de délégation de l'autorité, fondé sur l'asymétrie d'information, est en lien direct avec les arguments de Rajan et Zingales. En effet, la thématique du pouvoir de l'employé, fondé sur la spécificité de ses actifs humains, englobe sans difficulté la problématique de l'asymétrie informationnelle. L'information spécifique que détient l'agent dans le modèle d'Aghion et Tirole est assimilable, chez Rajan et Zingales, à un savoir tacite acquis au cours de la spécialisation : il est inclus dans les actifs humains spécifiques du subordonné. Par conséquent, ce modèle de délégation

${ }^{26}$ Cf. sous-section suivante. 
d'autorité rend bien compte d'un mécanisme organisationnel d'équilibration, et de stabilisation, entre l'autorité de l'employeur, mise en évidence par GHM, et le pouvoir limitatif de l'employé, souligné par Rajan et Zingales. L'autorité réelle chez Aghion et Tirole correspond donc au pouvoir limitatif de l'employé mis en avant par Rajan et Zingales. Enfin, la complémentarité et la cohérence entre ces trois modèles est d'autant plus forte qu'ils s'inscrivent dans le même cadre analytique, celui de la théorie des contrats incomplets.

La discussion précédente montre que l'on assiste à l'émergence d'une économie du pouvoir dans les organisations. Pourtant, au delà des aspects conflictuels de la relation d'autorité, la dimension coopérative de la relation d'emploi doit être aussi mise en lumière.

\section{II/ La coopération dans la relation d'emploi : l'acceptation de la relation d'autorité}

Deux contributions majeures tentent de rendre compte de l'autorité au sein de la firme en développant l'idée qu'il existe un contrat implicite entre l'employeur et l'employé, il s'agit d'une part des travaux de Williamson, et d'autre part d'un article de Kreps datant de 1990. Bien que le point de départ de ces deux approches soit relativement voisin, à savoir l'absence de référence à un quelconque pouvoir de la part de l'employeur, pouvoir issu de la possession des actifs non humains par l'employeur, le support théorique pour rendre compte de ce contrat implicite est néanmoins différent.

\section{A/ Le point de vue de Williamson : le concept de subordination volontaire}

On sait que chez Williamson, la référence au concept d'autorité intrafirme est récurrente car il constitue une pièce essentielle de sa problématique. En effet, pour cet auteur, l'autorité, en tant que mécanisme de coordination, permet de distinguer la firme des autres structures de gouvernement que sont les formes d'organisation hybrides et le marché (Williamson [1991]). Par ailleurs, l'autorité joue un rôle essentiel pour canaliser les éventuels comportements opportunistes (Williamson [1975]) et pour faciliter l'adaptation de la firme face aux perturbations de l'environnement. Dès son ouvrage de 1975, Williamson place ainsi l'autorité au cour de son analyse de la relation d'emploi et de la firme en reprenant les prémisses de l'analyse de Coase [1937] : «la hiérarchie implique généralement une relation supérieur-subordonné » (Williamson [1975], p. XV). De même, dans son ouvrage de 1985, Williamson, réaffirmant l'opposition marché / firme, définit de la manière suivante la "structure de gouvernance unifiée" - la firme- : «la transaction est retirée du marché et organisée à l'intérieur de la firme soumise à une relation d'autorité (intégration verticale)» (Williamson [1985], p. 75-76). Mais quel est le fondement de l'autorité intrafirme pour cet auteur ? Pour Williamson, l'autorité intrafirme repose en fait sur deux dispositifs, complémentaires mais 
différents ${ }^{27}$. D'une part, Williamson se rallie à la conception défendue notamment par Coase [1937], Simon [1951] et par Masten [1988], pour qui l'autorité trouve son origine dans la spécificité juridique du contrat de travail, conception que mus avons développée dans la section précédente. En effet, pour Williamson, les règles juridiques issues de l'environnement institutionnel constituent des ressources complémentaires des arrangements privés et elles représentent pour lui les "règles du jeu" (Williamson [1993], p. 113).

Mais, d'autre part, Williamson s'attache surtout, dans ses différents travaux, à montrer que l'employé, lorsqu'il rentre dans une organisation, adhère en fait aux objectifs de cette organisation, et ce sous la forme d'un contrat implicite. En ce sens, il semble que Williamson reprend largement à son compte la conception de l'autorité et de la relation d'emploi proposée par Barnard dans son ouvrage de 1938 (Williamson [1990]). En effet, Williamson note dans l'introduction de son ouvrage de 1975 : «la relation d'emploi est généralement associée à une subordination volontaire » (Williamson [1975], p. XV). Le fait de mettre en avant ce concept de subordination volontaire est donc fondamental car dans l'esprit de Williamson, les individus arbitreraient entre intégrer une hiérarchie ou choisir un autre mode d'organisation économique, par exemple un groupe de pairs. S'ils choisissent la première branche de l'alternative, c'est qu'en fin de compte, en acceptant librement- l'autorité et donc une relation hiérarchique, ils augmentent leur niveau de satisfaction. Autrement dit, alors que pour Hart, l'autorité s'appuie sur la menace exercée en permanence par l'employeur de retirer à l'employé les actifs non humains en sa possession, pour Williamson, l'autorité résulte d'une adhésion librement consentie par l'individu, qui considère d'emblée que la relation hiérarchique est bénéfique pour lui. Sans le dire vraiment explicitement, Williamson semble ainsi adhérer à la théorie de "l'équilibre de l'organisation" développée par Barnard dans son ouvrage de 1938, théorie selon laquelle les individus, pour accepter l'autorité, feraient un calcul entre la contribution fournie et la rétribution perçue, théorie reprise par la suite par Simon [1947] et par March et Simon [1958] ${ }^{28}$. Ce point de vue est crucial car dès lors, la mise en place de sanctions et d'incitations pour obtenir "l'obéissance" des individus, même si elle n'est pas totalement absente, est reléguée en arrière-plan de la problématique de Williamson, puisque les individus, rentrant dans une organisation, se placent volontairement en situation de subordination, c'est-àdire acceptent les commandements et les ordres, certes à l'intérieur d'une "zone d'acceptation". Cette dernière expression, reprise également de Barnard, accentue par ailleurs le caractère volontaire de la subordination. Les incitations sont alors conçues davantage pour obtenir une coopération maximale

\footnotetext{
27 Voir sur ce point un article récent de Bernard Baudry [1999].

28 Simon développe à nouveau sa conception de la relation d'emploi dans son article de 1991.
} 
de la part des salariés qu'une obéissance stricte. Au total, l'autorité intrafirme résulte bien en partie d'un contrat implicite entre l'employeur et l'employé : l'individu obéit tant qu'il considère que l'employeur, de son côté, crée les conditions de cette acceptation, notamment en versant une rétribution perçue comme suffisante par l'employé, et en ne sortant pas de la zone d'acceptation définie ex ante.

Cette analyse est d'autant plus intéressante que Williamson envisage le cas où l'employeur serait tenté de remettre en cause ce contrat implicite, par exemple en voulant capter la rente issue des actifs humains spécifiques engagés par l'employé.

En ce sens, Williamson ne développe pas une vision naï ve, voire angélique de la relation d'emploi. La conception de l'autorité qu'il développe n'exclut en effet nullement les possibilités de conflit entre un employeur et ses employés, et ce d'autant plus que pour lui, la relation d'emploi n'est pas symétrique. En effet, dans son ouvrage de 1985, il note que "le travailleur n'est qu'un parmi une multitude et l'employeur peut et obtiendra des avantages stratégiques en faisant un exemple sur un ou plusieurs travailleurs, donnant une leçon à tous. L'argument de la symétrie entre l'employeur et l'employé est donc pour lui non fondée car elle ignore cette "disparité fondamentale" (Williamson [1985], p. 260).

En revanche, Williamson ne relie pas l'autorité au pouvoir, contrairement à Hart, bien qu'il pose en revanche clairement la question de l'abus de l'autorité par un employeur qui adopterait alors un comportement de "prédateur" (ibid., p. 262) ${ }^{29}$. Il indique alors que plusieurs dispositifs encadrent un tel comportement : tout d'abord la présence des syndicats, qui ont pour fonction de maintenir les termes du contrat implicite, ensuite la capacité des salariés à pénaliser l'employeur en choisissant une coopération minimale au lieu d'une coopération maximale, enfin la perte de réputation d'un tel employeur, qui aurait alors de la difficulté à recruter de nouveaux salariés.

Le fait que pour Williamson la relation employeur / employé soit déséquilibrée "dès le départ" ne semble pas contradictoire avec l'accent placé sur l'aspect volontaire de la subordination. Simplement, ces deux thématiques interviennent à deux moments différents de la relation d'emploi : ex ante, l'individu adhère librement aux objectifs de l'organisation, donc accepte d'obéir, mais cette acceptation sera remise en cause si en cours du contrat, l'employeur abuse de sa position de domination. On retrouve également cet aspect consensualiste quand Williamson indique que les employeurs et les employés ont tout intérêt à s'entendre lorsque des actifs spécifiques humains sont en jeu.

\footnotetext{
${ }^{29}$ Notons que cette question ne rentre pas dans la problématique de Hart.
} 
La problématique de Williamson est donc finalement très riche et complète selon nous la perspective adoptée par la théorie des droits de propriété. En effet, si cette dernière envisage l'aspect coercitif du contrat de travail par l'intermédiaire de la possession par l'employeur des actifs non humains, Williamson, en mettant en partie l'accent sur l'adhésion librement consentie des individus à l'autorité de l'employeur dans une organisation donnée, insiste au contraire sur l'aspect positif de la relation d'emploi, à savoir la volonté d'adhérer ex ante aux objectifs de l'organisation. Par ailleurs, Williamson envisage sans ambiguï té la question de l'opportunisme de l'employeur, qui serait éventuellement tenté d'abuser de l'autorité qui lui a été -en partie- librement transférée. Or, les travaux de Hart éludent cette question, les individus obéissant de manière quasi mécanique.

En ce sens, comme nous allons maintenant le voir, le travail de Kreps se situe dans la lignée de celui de Williamson, puisque Kreps approfondit le concept de réputation, cette dernière constituant en définitive un dispositif facilitant l'adhésion des individus à l'organisation et donc à l'autorité, compte tenu de l'incomplétude du contrat de travail.

\section{$\underline{\text { B/ Réputation et confiance : l'analyse de Kreps }}$}

Comme Williamson, Kreps considère que l'autorité entre un employeur et un employé résulte d'un contrat implicite passé entre ces deux individus, et non pas de la possession par l'employeur des actifs non humains. Kreps note tout d'abord qu'il existe deux types de transactions, d'une part des transactions "spécifiées" et d'autre part des transactions "hiérarchiques" (Kreps [1990], p. 99) ${ }^{30}$. Dans une transaction spécifiée, comme son nom l'indique, tous les termes du contrat peuvent être décrits à l'avance. En revanche, dans les transactions hiérarchiques, qui concernent la mise en åvre de la relation d'emploi, certains termes ne sont pas spécifiées. Ce qui est spécifié ex ante, c'est le fait qu'une partie dispose de l'autorité, à l'intérieur de certaines limites, de déterminer ex post l'exécution du contrat, lorsque des contingences surviennent. Autrement dit, dans ce type de transactions, comme le note Kreps, «la partie hiérarchiquement supérieure, explicitement ou implicitement, a le droit de diriger la partie inférieure » (ibid., p. 113). La question que pose Kreps est alors la suivante : «pourquoi quelqu'un entrerait-il volontairement dans un tel contrat à la position inférieure?»(ibid.). Autrement dit, comment expliquer que les employés laissent à l'employeur le droit de spécifier ex post les arrangements nécessaires à la réalisation du contrat ? Or, pour Kreps, si les employés acceptent l'autorité, c'est parce qu'ils pensent que cette autorité sera utilisée impartialement, équitablement (“fairly”). Il convient alors de s'interroger sur le fondement de cette "croyance" (ibid., p. 92) qui implique que l'employé accorde sa "confiance" à l'employeur.

\footnotetext{
30 Comme le note lui-même Kreps, cette dichotomie renvoie à celle effectuée par Simon dans son article de 1951.
} 
En effet, et c'est là un apport significatif de Kreps, laisser au supérieur hiérarchique le droit de compléter le contrat - lui confier l'autorité- en cours de réalisation peut donner lieu à des abus de la part de ce supérieur ${ }^{31}$. Pour étayer sa démonstration, Kreps présente alors dans un premier temps le jeu suivant, qu'il dénomme le jeu de la confiance.

Le jeu de la confiance (Kreps [1990], p. 100)

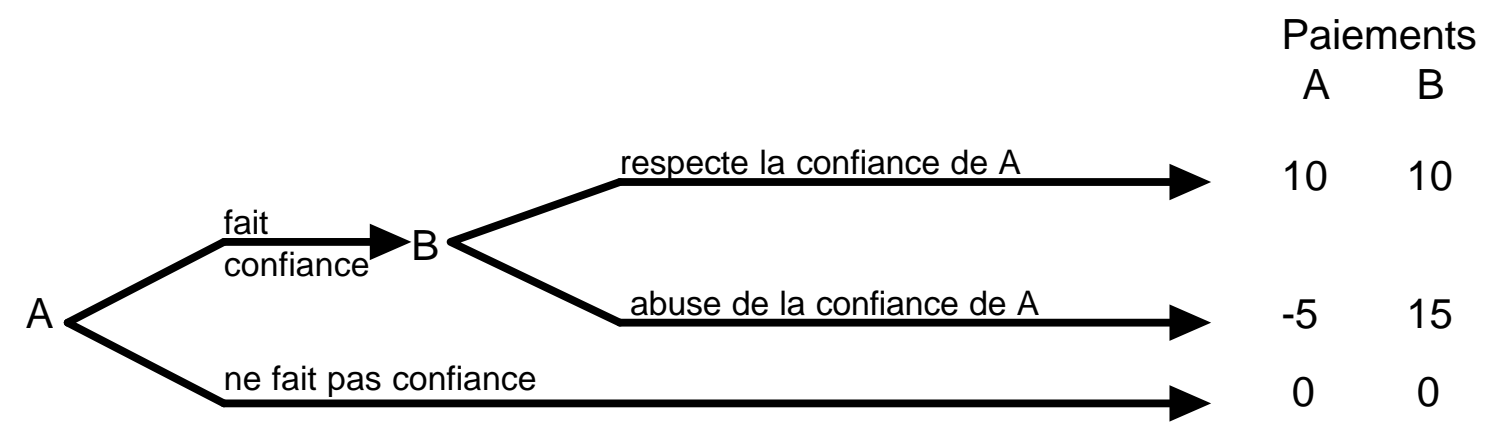

On constate que si le jeu est joué une seule fois et de manière non coopérative, A ne fera alors pas confiance à B.

Kreps donne ensuite l'exemple suivant, caractéristique d'une transaction hiérarchique. Un employeur B embauche un employé A pour réaliser une certaine tâche, réalisation qui peut être soit facile soit difficile. Si la tâche est facile, un paiement de 3\$ est suffisant pour l'employé et si elle est difficile, un paiement de $13 \$$ est exigé. Kreps fait alors l'hypothèse -cruciale- que le résultat du contrat est observable mais non vérifiable. Dans ces conditions, si l'employeur et l'employé traitent une seule fois, la transaction échouera, comme dans le jeu de la confiance présenté auparavant. En effet, l'employeur abusera de la confiance qui lui est octroyée par l'employé, c'est-àdire effectuera un paiement de 3\$, même si h tâche est en fin de compte difficile. Mais Kreps considère alors qu'en fait, l'employeur B ne traite pas qu'avec un seul employé A, mais avec une séquence de A, et dans ces conditions des employés potentiels futurs refuseront de traiter avec B si ce dernier abuse de la confiance qui lui est accordée. La réputation de l'employeur, qui est souillée s'il trahit la confiance, se trouve dans ces conditions au coeur de la transaction hiérarchique et du contrat implicite qui lie le supérieur hiérarchique et son subordonné. La réputation constitue donc bien le support d'un contrat de confiance implicite auto-renforçant entre l'employeur et l'employé ${ }^{32}$. Cette démonstration permet ensuite à Kreps de donner la réponse posée au début de son raisonnement : «pourquoi quelqu'un entrerait-il volontairement dans un tel contrat à la position inférieure? Ce

\footnotetext{
31 On retrouve donc ici une partie de la problématique de Williamson.
} 
peut être parce que le pire qui puisse arriver est suffisamment bon pour que la transaction en vaille la peine. Mais lorsque la partie supérieure a une réputation à sauvegarder ou à accroître, une réputation qui concerne la façon dont cette partie exerce l'autorité, alors la partie inférieure n'a pas besoin de présumer du pire. Cette dernière peut compter sur la partie supérieure pour être fidèle à un contrat implicite conforme à son propre intérêt » (ibid., p. 113).

Néanmoins, comme le souligne Kreps lui-même, la réputation reste un mécanisme de coordination fragile, dans la mesure où elle nécessite la réunion de plusieurs conditions. Comme l'écrit François Eymard-Duvernay, l'établissement d'une réputation «suppose des outils de diffusion des informations. Compte tenu de ce coût, le passage à des transactions entre des individus en grand nombre n'est pas immédiat. Bien souvent, la réputation ne sera effective que sur des réseaux individuels limités » (Eymard-Duvernay [1994], p. 316).

Par ailleurs, pour que le mécanisme de la réputation puisse fonctionner, les contractants doivent être capables de vérifier les engagements conclus ex ante. Eymard-Duvernay note à juste titre qu'«il faut que l'on puisse déterminer ex post sans ambiguïté si la partie susceptible de faire défaut a coopéré ou non » (ibid., p. 315).

Enfin, Kreps envisage également les difficultés de coordination, et donc le rôle de l'autorité, lorsque des événements non envisagés ex ante surviennent ex post. Pour lui, la présence d'une culture d'entreprise, matérialisée par des règles issues d'un processus d'apprentissage, sert de point focal auquel les membres d'une organisation pourraient se coordonner pour faire face à ces événements. Cette culture d'entreprise est censée donner aux individus en situation hiérarchique inférieure une idée sur la manière dont l'organisation réagira dans des circonstances particulières. Elle est donc non seulement un outil de coordination, mais également le moyen de coordonner l'exercice de l'autorité hiérarchique. En effet, la mise en oæivre et le respect de ces règles par les personnes qui agissent au nom de la firme, les supérieurs hiérarchiques, constitueraient un dispositif de contrôle palliant la non-observabilité de leurs actions et de leurs résultats.

En définitive, cette deuxième partie de l'argumentation de Kreps semble moins convaincante 33 que sa démonstration du mécanisme de la réputation comme fondement de l'acceptation de l'autorité, de par sa très grande généralité et sur l'absence de précision sur le processus d'apprentissage et du type de règles qui émerge. Par ailleurs, il faut souligner le flou relatif qui entoure la nature de l'autorité que retient Kreps. En effet, on ne sait pas toujours si cet auteur fait référence à l'autorité formelle où à l'autorité réelle. Par exemple, quand il définit la relation d'emploi et donc le contrat de travail, il

32 Ce raisonnement permet à Kreps de souligner qu'il est plus rationnel que ce soit le supérieur hiérarchique qui dispose de l'autorité.

33 Kreps reconnaît lui-même que son exposé est en partie exploratoire. 
traite clairement de l'autorité formelle, et il s'appuie alors à juste titre sur l'article de Simon [1951]. En revanche, dans d'autres passages, il s'intéresse à la relation inférieur / supérieur hiérarchique, et ce dernier n'est pas nécessairement l'employeur, ce qui renvoie de ce fait plutôt au mécanisme de l'autorité réelle.

Malgré ces réserves, le traitement de la question posée par Kreps sur l'acceptation de l'autorité -et donc sur son abus éventuel par l'employeur- est pertinent, et approfondit de notre point de vue fructueusement le point de vue de Williamson. En effet, ces deux analyses montrent que l'individu, rentrant dans une organisation, accepte librement l'autorité dans la mesure où il sait que plusieurs dispositifs, dont la réputation de l'employeur, vont garantir le contrat implicite auquel il adhère.

Les contributions de Williamson et de Kreps s'avèrent donc finalement complémentaires de celles que nous avons développées précédemment. En effet, alors que Hart place l'accent sur la capacité de l'employeur, en cours de contrat, à obtenir l'obéissance des employés par l'intermédiaire de la menace d'exclusion des actifs non humains, Williamson et Kreps montrent l'acceptation volontaire des salariés à l'autorité, donc sans recours à la menace, et cette acceptation existe ex ante, lors de l'entrée des individus dans une organisation.

\section{Conclusion : des approches complémentaires}

Les approches présentées dans cette contribution éclairent chacune un aspect différent de la relation d'autorité. Ainsi que nous l'avons mis en évidence tout au long de cet article, ces différences témoignent davantage d'une complémentarité que d'une opposition. Il se dégage de notre analyse l'idée centrale que l'autorité de l'employeur doit être appréhendée en termes de degrés. Il convient en effet de comprendre comment la réalité se situe nécessairement entre deux pôles : d'une part le “degré 0" de l'autorité, situation où l'employeur n'a strictement aucune prise sur ses subordonnés, et, d'autre part, le "degré 1" où l'employé ne dispose d'aucune autonomie. L'autorité dans l'organisation doit donc être pensée comme un savant mélange d'autonomie et de contrôle. Comment ce mélange s'opère-t-il ? Comme nous pensons l'avoir montré, il résulte de la conjonction de trois forces principales agissant selon leur propre logique dans des directions différentes : les ressources de l'employeur, les actifs spécifiques humains et la coopération.

Ainsi que l'indique le schéma ci-dessous, l'employeur peut s'appuyer sur deux types différents de ressources pour asseoir son autorité. D'une part, la propriété des actifs non-humains (Hart) fonde cette autorité en donnant à l'employeur les moyens réels de l'exercer sur le salarié par la menace crédible de licenciement (1). D'autre part, l'environnement institutionnel (Masten) vient conforter et consacrer cette autorité par le droit en inscrivant dans la loi la subordination juridique de 
l'employé (2). Celui-ci, quant à lui, ne subit pas passivement cette autorité, il dispose de certaines ressources pour la limiter. Son pouvoir limitatif (Rajan et Zingales), appelé aussi autorité réelle (Aghion et Tirole), est fondé sur la spécificité de ses actifs humains (3). Toutefois, il ne s'agit pas d'un pur rapport de forces. Les principes de délégation de l'autorité établissent en effet un mécanisme interne à la relation d'emploi d'équilibration et de stabilisation du pouvoir de l'employeur et de l'employé. Enfin, l'autorité ne repose pas uniquement sur la contrainte. Son acceptation volontaire par l'employé, lors de son entrée dans l'organisation, et la confiance qui s'instaure au fil de la relation montrent l'importance de la coopération (4).

Le degré de l'autorité $A$ de l'employeur sur l'employé est donc une fonction croissante des ressources $R_{e}$ disponibles pour l'employeur : essentiellement, la facilité plus ou moins grande de licenciement. Inversement, plus les actifs humains $R_{S}$ de l'employé seront spécifiques, plus son autonomie sera importante et moins le degré d'autorité sera élevé. Enfin, la coopération $C$ agit positivement sur $A$ : plus $C$ est forte moins l'employeur sera contesté dans ses décisions. Il convient ici de distinguer autorité et autoritarisme. L'autoritarisme est uniquement une fonction inverse de la coopération. Toutes choses égales par ailleurs, moins la coopération est prononcée, plus l'employeur doit recourir à la contrainte et à la menace pour que ses ordres soient appliqués. Un degré important d'autorité est donc tout à fait conciliable avec des rapports peu autoritaires si le niveau de coopération est élevé. 
Principaux déterminants du degré de l'autorité de l'employeur

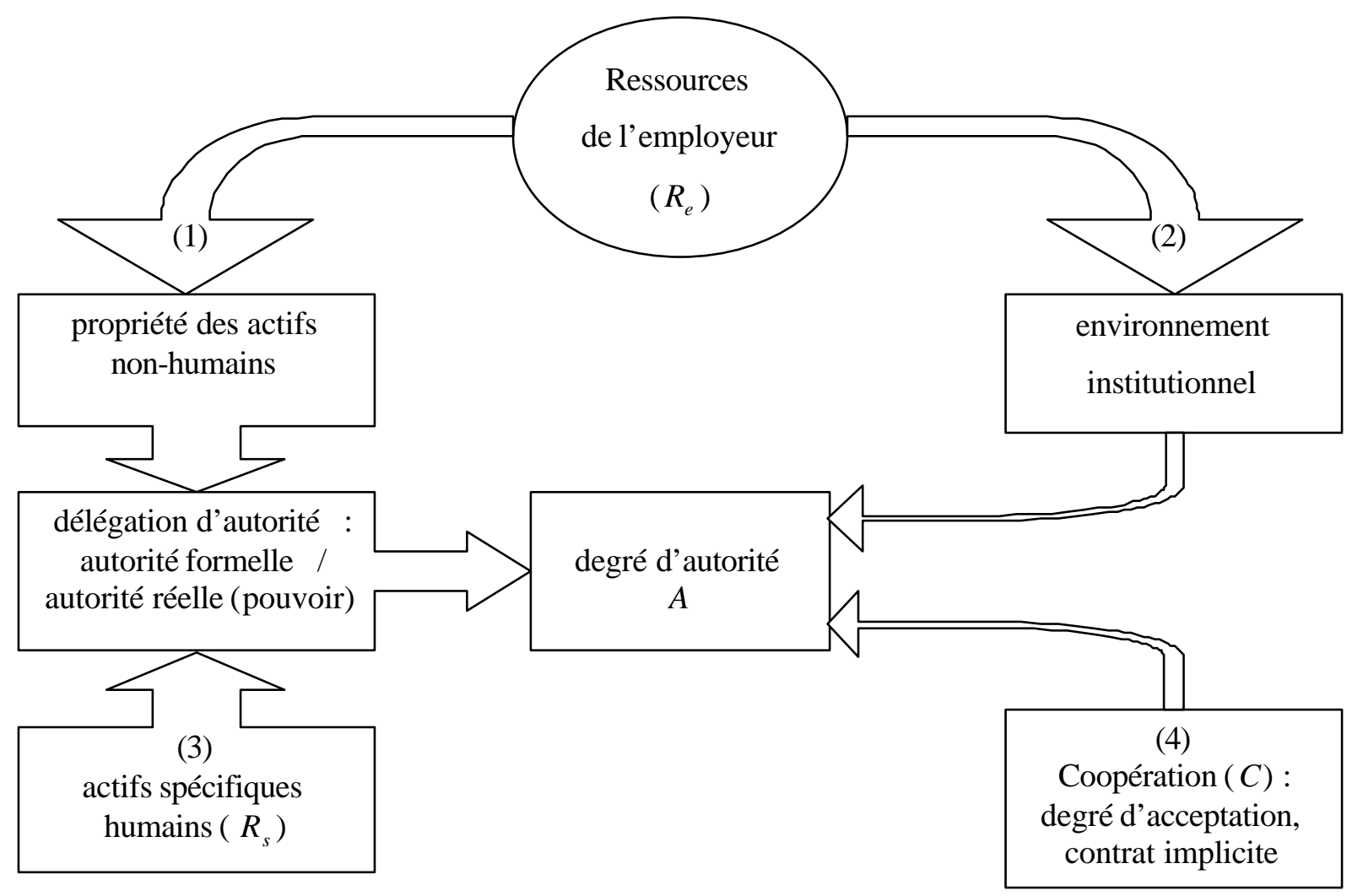

Soit $A$, le degré de l'autorité de l'employeur, défini comme suit $\quad: A=\Phi\left(R_{e}, R_{s}, C\right)$, avec $\frac{\partial \Phi}{\partial R_{e}}, \frac{\partial \Phi}{\partial C}>0$ et $\frac{\partial \Phi}{\partial R_{s}}<0$

\section{REFERENCES}

AgHION, Philippe \& Jean TrROLE [1997] "Formal and real authority in organizations", Journal of Political Economy, 105 (1), pp. 1-29.

Alchian, Armen A. \& Harold DEMSETZ [1972] "Production, Information Costs, and Economic Organisation", American Economic Review, 62, december, pp.777-795.

ARROW, Kenneth [1976] Les limites de l'organisation, PUF, Paris (1974).

BAKER, George, Robert GIBBONS \& Kevin J. MURPHY [1999] "Informal authority in organizations", Journal of Law, Economics, and Organization, 15 (1), pp. 56-73. 
BARNARD, Chester [1938] The functions of the executive, Harvard University Press.

BAUDRY, Bernard [1999] "L'apport de la théorie des organisations à la conception néoinstitutionnelle de la firme : une relecture des travaux de O. E. Williamson", Revue Économique, janvier, vol. 50, $\mathrm{n}^{\circ} 1$, pp. 45-69.

COASE, Ronald [1937] “The Nature of the Firm”, Economica, 4, november, pp. 386-405 (traduit en français dans Revue Française d'Economie, 1987 vol. II/1, p. 133-163).

EYMARD-DUVERNAY, François [1994] “Coordination des échanges par l'entreprise et qualité des biens", dans Analyse économique des conventions, A. Orléan (éd.), PUF, Paris, p. 307-334.

GOLDBERG, Victor [1976] "Regulation and administered contracts", Bell Journal of Economics, 7, pp. 426-448.

Grossman, Sanford J. \& Oliver D. HART [1986] "The costs and benefits of ownership: a theory of vertical and lateral integration", Journal of Political Economy, 94 (4), pp. 691-719.

HART, Oliver D. [1995] Firms, contracts, and financial structure Oxford University Press, Clarendon Lectures in Economics.

HART, Oliver D. \& John MOORE [1990] "Property rights and the nature of the firm", Journal of Political Economy, 98 (6), pp. 1119-1158.

HOLMSTRÖM, Bengt [1999] "The firm as a subeconomy”, Journal of Law, Economics, \& Organization 15 (1), pp. 74-102.

HOLMSTRÖM, Bengt \& John ROBERTS [1998] "The boundaries of the firm revisited", Journal of Econmic Perspectives, 12 (4), pp. 73-94.

JeAmmaud, Antoine, Le Friant, Martine \& LyON-CAEN, Antoine [1998] "L'ordonnancement des relations du travail”, Recueil Dalloz, Chron. 359.

Jensen, M. C. \& Meckling, W. H. [1976] "Theory of the Firm: Managerial Behavior, Agency Cost, and Ownership Structure”, Journal of Financial Economics, 3, pp. 305-360.

KIRAT, Thierry [1998] "Économie et droit: de l'analyse économique du droit à de nouvelles alliances?", Revue Économique, 49 (4), 1057-1087.

KLEIN, Benjamin, Robert G. CRAWFORD \& Armen A. ALCHIAN [1978] "Integration, appropriable rents, and the competitive contracting process", Journal of Law \& Economics, XXI (2), pp. 297326.

KREPS, David [1990] "Corporate culture and economic theory" in Perspectives on political economy, J. ALT et K. SHEPSLE (eds), Cambridge University Press, p. 90-143.

MALCOMSON, James M. [1997] "Contracts, hold-up, and labor markets", Journal of Economic Literature, XXXV, december, pp. 1916-1957.

MARCH, James \& Herbert SimON [1958] Organizations, New York : Wiley. 
MASTEN, Scott [1991] "A legal basis for the firm", in The nature of the firm; origins, evolution and development, O. Williamson and S. Winter (eds), Oxford University Press, p. 196-212 (première publication dans Journal of Law, Economics and Organization, 4, spring 1988).

MENARD, Claude [1994] "Organizations as coordinating devices”, Metroeconomica, vol. 45, n 3, p. 224-247.

RAJAN, Raghuram \& Luigi ZNGALES [1998] "Power in a theory of the firm", Quarterly Journal of Economics, may, pp. 387-432.

Review of Economic Studies [1999] "Special issue: contracts", 66 (1), n 226.

SHAPIRO, Carl \& Joseph E. STIGLITZ [1984] "Equilibrium unemployment as a worker discipline device", American Economic Review, 74, pp. 433-444.

SIMON, Herbert [1947] Administrative Behavior. A Study of Decision-Making Processes in Administrative Organization, The free Press, New York.

SIMON, Herbert [1951] "A formal theory of the employment relationship", Econometrica, 19 (3), p. 293-305.

SIMON, Herbert [1991] "Oganizations and Markets", Journal of Economic Perspectives, 5 (2), spring, pp. 25-44.

TIROLE, Jean [1999] "Incomplete contracts: where do we stand ?", Econometrica, 67 (4), pp. 741781.

WiLliamson, Oliver E. [1975] Markets and Hierarchies, The Free Press: New York.

WiLliamSON, Oliver E. [1985] The economic institutions of capitalism, Free Press, New York.

WiLliAMSON, Oliver E. [1990] "Chester Barnard and the incipient science of organization”, in Organization and theory, From Chester Barnard to the present and beyond, O. Williamson (ed), Oxford University Press, p. 172-206.

WiLliamson, Oliver E. [1991] "Comparative economic organization : the analysis of discrete alternative", Administrative Science Quaterly, vol. 36, p. 269-296. 